\title{
Association of NLRP1 and NLRP3 Polymorphisms with Psoriasis Vulgaris Risk in the Chinese Han Population
}

\author{
Pei Yu $\left(\mathbb{D},{ }^{1}\right.$ Siyu Hao, ${ }^{1}$ Hewei Zheng, ${ }^{2}$ Xueying Zhao, ${ }^{2}$ and Yuzhen Li ${ }^{1}{ }^{1}$ \\ ${ }^{1}$ Department of Dermatology, The Second Affiliated Hospital of Harbin Medical University, Harbin 150081, China \\ ${ }^{2}$ Department of Bioinformatics, Harbin Medical University, Harbin 150001, China \\ Correspondence should be addressed to Yuzhen Li; liyuzhen@medmail.com.cn
}

Received 12 September 2017; Revised 29 January 2018; Accepted 13 February 2018; Published 3 April 2018

Academic Editor: Ruben Queiro-Silva

Copyright (c) 2018 Pei Yu et al. This is an open access article distributed under the Creative Commons Attribution License, which permits unrestricted use, distribution, and reproduction in any medium, provided the original work is properly cited.

\begin{abstract}
Aim. To clarify the association between the single nucleotide polymorphisms (SNPs) in the NLRP1 and NLRP3 and Psoriasis Vulgaris (PsV) in the Chinese Han population. Methods. We genotyped eight SNPs, four from NLRP1 (rs8079034, rs11651270, rs11657747, and rs878329) and NLRP3 (rs7512998, rs3806265, rs10754557, and rs10733113) each in 540 patients with PsV and 612 healthy controls in the Chinese Han population using an improved multiplexed ligation detection reaction (iMLDR) method. The genotype and haplotype frequencies were analyzed using a case-control study design. Results. We identified two SNPs, rs3806265 and rs10754557, in NLRP3 that were significantly associated with PsV. The genotype distribution of the rs 3806265 SNP was significantly different between cases and controls $(p=0.0451$; OR $=0.791 ; 95 \% \mathrm{CI}=0.627-0.998)$. In the recessive model, the genotype distribution of the rs10754557 SNP was significantly different between cases and controls ( $p=0.0344$; OR $=1.277 ; 95 \% \mathrm{CI}=0.987-1.652)$. The haplotype analysis of rs3806265 and rs10754557 also presented a significant association of TA haplotype with PsV $\left(\chi^{2}=4.529\right.$; $p=0.033$ ). Conclusion. NLRP3 may play a role in PsV susceptibility in the Chinese Han population.
\end{abstract}

\section{Introduction}

Psoriasis is a common, immune-mediated, chronic inflammatory skin disease that causes red, flaky patches of skin [1], which affects approximately $2 \%$ of the general population [2]. Although its etiology is not fully understood, psoriasis has been established as a complex and multifactorial disease related to environmental and genetic factors [3]. The number of identified loci for European populations has increased to 63 and many functional networks and gene regulatory signals associated with psoriasis have been revealed by the largest GWAS meta-analysis for psoriasis patients to date in European populations [4]. The most accurate genetic prediction model to date involving 14 psoriasis susceptibility loci shows that these loci have the discriminating potential, being also associated with family history and age of onset [3]. These findings provide new insights into the pathogenesis of psoriasis and have greatly enhanced our understanding of the disease mechanisms. However, a substantial portion of information regarding the heritability of the disease remains unknown and further studies are required [3].
Members of the nucleotide-binding oligomerization domain-like receptor (NLR) family are the major components of inflammasomes [5], which are intracellular complexes assembled by proinflammatory caspase, NLR family members containing pyrin domain (NLRP), and the adaptor molecule apoptosis-associated speck-like protein [6]. Inflammasomes play a vital role in the maturation and secretion of proinflammatory cytokines [7], including interleukin- $1 \beta[6,8,9]$, which cause a wide variety of biological effects associated with infection, inflammation, and autoimmune processes by triggering the adaptive immune response [10]. NLRP1 and NLRP3 are the two most wellstudied inflammasome-forming NLR family members [9]. These proteins might represent important links between the innate and the adaptive immune system in chronic inflammatory diseases [11] and have been strongly implicated in the pathogenesis of psoriasis $[8,9]$.

NLRP1 and NLRP3 expression has been observed in the lesional as well as nonlesional psoriatic epidermis [11]. The NLRP1 mRNA expression level was reported to be increased 
in the peripheral blood of patients with psoriasis [9], and the NLRP3 expression level was reported to be increased in the leucocytes and keratinocytes of psoriasis lesions, but not in the parakeratotic cells on the surface of psoriasis lesions [12]. The NLRP3 inflammasome complex and protein components were detected in both cultured and primary human keratinocytes $[12,13]$. Therefore, NLRP1 and NLRP3 may play roles in the dysregulated innate immune response that is characteristic of psoriasis.

Different autoimmune diseases share susceptibility loci [14]. For example, the association analysis by $\mathrm{Li}$ et al. identified two common susceptibility loci shared by psoriasis and systemic lupus erythematosus (SLE) in the Chinese Han population [14]. Single nucleotide polymorphisms (SNPs) in the NLRP1 have been implicated in several complex autoimmune conditions such as juvenile idiopathic arthritis [15], rheumatoid arthritis (RA), vitiligo, autoimmune Addison's disease, and Type I Diabetes [16]. Moreover, NLRP3 gene polymorphisms have been explored as possible predisposing factors for development of a variety of diseases associated with autoimmune and/or inflammatory responses, such as RA, Crohn's disease, SLE, celiac disease, AIDS, Alzheimer's disease, abdominal aortic aneurysms [17], atopic dermatitis $[17,18]$, and malignant melanoma [19]. NLRP1 and NLRP3 polymorphisms have been associated with psoriasis in the European population $[9,12]$; however, their association with psoriasis in other populations remains unexplored. Psoriasis has a considerable ethnic variation. Allelic and/or locus heterogeneity exists between different populations $[5,20]$. Sun et al. identified six susceptibility loci in the Chinese population by adopting a multistage analysis strategy for several large samples from a Chinese and European population [21], which fully illustrated this point of view. Therefore, it is important to determine the genetic association between SNPs in NLRP1 and NLRP3 and susceptibility of PsV in the Chinese population. Accordingly, the aim of the current study was to investigate the association between SNPs in NLRP1 and NLRP3 and susceptibility of PsV in the Chinese Han population.

\section{Materials and Methods}

2.1. Study Subjects. In total, 540 patients diagnosed with PsV and 612 healthy individuals were recruited from outpatients of the 2nd Affiliated Hospital of Harbin Medical University (January 2013 to April 2015) in this study. All patients and healthy controls in this study were of Han Chinese descent (including first-, second-, and third-degree relatives). All the patients with PsV had at least two skin lesions. Diagnosis was based on evaluation of clinical features by at least two trained dermatologists at the 2nd Affiliated Hospital of Harbin Medical University and confirmed by skin biopsy at the same institution. The healthy controls were randomly recruited from patients visiting the same hospital for routine health examinations, who were confirmed to not be relatives of the patient group. Self-reported information from a standard questionnaire was used to collect demographic and other characteristics (severity, age of onset, and family history, including three levels of relatives) from the patients and to exclude controls with psoriasis or a family history of psoriasis, including three levels of relatives, or any other systemic, infectious, autoimmune, atopic, or malignant disease. None of the patients had arthritis.

All participants provided written informed consent. The study protocol was approved by the ethics committee of the 2nd Affiliated Hospital of Harbin Medical University and was conducted in full accordance with the Ethics Guidelines of the 1975 Declaration of Helsinki.

2.2. DNA Extraction. Peripheral venous blood samples $(2 \mathrm{~mL})$ were collected from each participant in tubes containing ethylenediaminetetraacetic acid. Genomic DNA for genotyping was prepared using standard extraction procedures (QIAamp DNA Blood Mini Kit, Qiagen, Germany) according to the manufacturer's recommendations and stored at $-20^{\circ} \mathrm{C}$.

2.3. SNPs Selection. We refer to the method described by Zhao et al. to find reasonable SNPs [22]. Data on SNP loci for complete NLRP1 and NLRP3 sequences were obtained from the dbSNP database (https://www.ncbi.nlm.nih.gov/ projects/SNP/), and SNP reference genotype data were retrieved from the NCBI HapMap database. First, we filtered all SNPs according to a HapMap SNP minor allele frequency $\geq 0.05$ for the Han Chinese in Beijing (HCB) population. We then preferentially selected the SNPs, namely, rs8079034, rs11651270, rs11657747, rs878329, rs7512998, and rs10733113, according to their linkage relationship, frequencies, and positions, and their reported associations with immune and inflammatory disease. Following comprehensive analysis of the experimental conditions for genotyping, we ultimately selected four SNPs in NLRP1 (rs8079034, rs11651270, rs11657747, and rs878329) and four SNPs in NLRP3 (rs7512998, rs3806265, rs10754557, and rs10733113) for our analysis. The specific process for selection is schematically represented by the SNP-screening flow chart (Figure 1).

2.4. Genotyping. Genotyping was performed using the iMLDR technique developed by Genesky Biotechnologies Inc. (Shanghai, China) as described previously [23].

Multiplex polymerase chain reaction (PCR) was used to amplify fragments including these eight SNP sites (see Tables S1 and S2 for the primer information). The $20 \mu \mathrm{L}$ PCR system consisted of $1 \mathrm{U}$ HotStart Taq DNA polymerase (Qiagen, Germany), 1x GCI buffer, $3.0 \mathrm{mM} \mathrm{Mg}^{2+}, 1 \mu \mathrm{L}$ primer mixture, $20 \mathrm{ng}$ genomic DNA, and $0.3 \mathrm{mM}$ dNTP (Generay Biotech). The PCR cycling program was as follows: $95^{\circ} \mathrm{C}$ for $2 \mathrm{~min}$; 11 cycles of $94^{\circ} \mathrm{C}$ for $20 \mathrm{~s}$, decreasing to $65^{\circ} \mathrm{C}$ at $0.5^{\circ} \mathrm{C}$ increments per cycle for $40 \mathrm{~s}$, and $72^{\circ} \mathrm{C}$ for $1.5 \mathrm{~min} ; 24$ cycles of $94^{\circ} \mathrm{C}$ for $20 \mathrm{~s}, 59^{\circ} \mathrm{C}$ for $30 \mathrm{~s}$, and $72^{\circ} \mathrm{C}$ for $1.5 \mathrm{~min}$; and a final extension at $72^{\circ} \mathrm{C}$ for $2 \mathrm{~min}$, followed by holding at $4^{\circ} \mathrm{C}$. The products were purified via digestion of $5 \mathrm{U}$ shrimp alkaline phosphatase (Promega) and $2 \mathrm{U}$ exonuclease I at $37^{\circ} \mathrm{C}$ for $1 \mathrm{~h}$, followed by $75^{\circ} \mathrm{C}$ for $15 \mathrm{~min}$. The ligation reaction was carried out in $10 \mu \mathrm{L}$ of a mixture comprising $1 \mu \mathrm{L} 10 \mathrm{x}$ ligation buffer, $0.25 \mu \mathrm{L} 80 \mathrm{U} / \mu \mathrm{L}$ Taq DNA ligase (New England Biolabs), $0.4 \mu \mathrm{L} 5^{\prime}$ ligation primer mixture $(1 \mu \mathrm{M})$, 


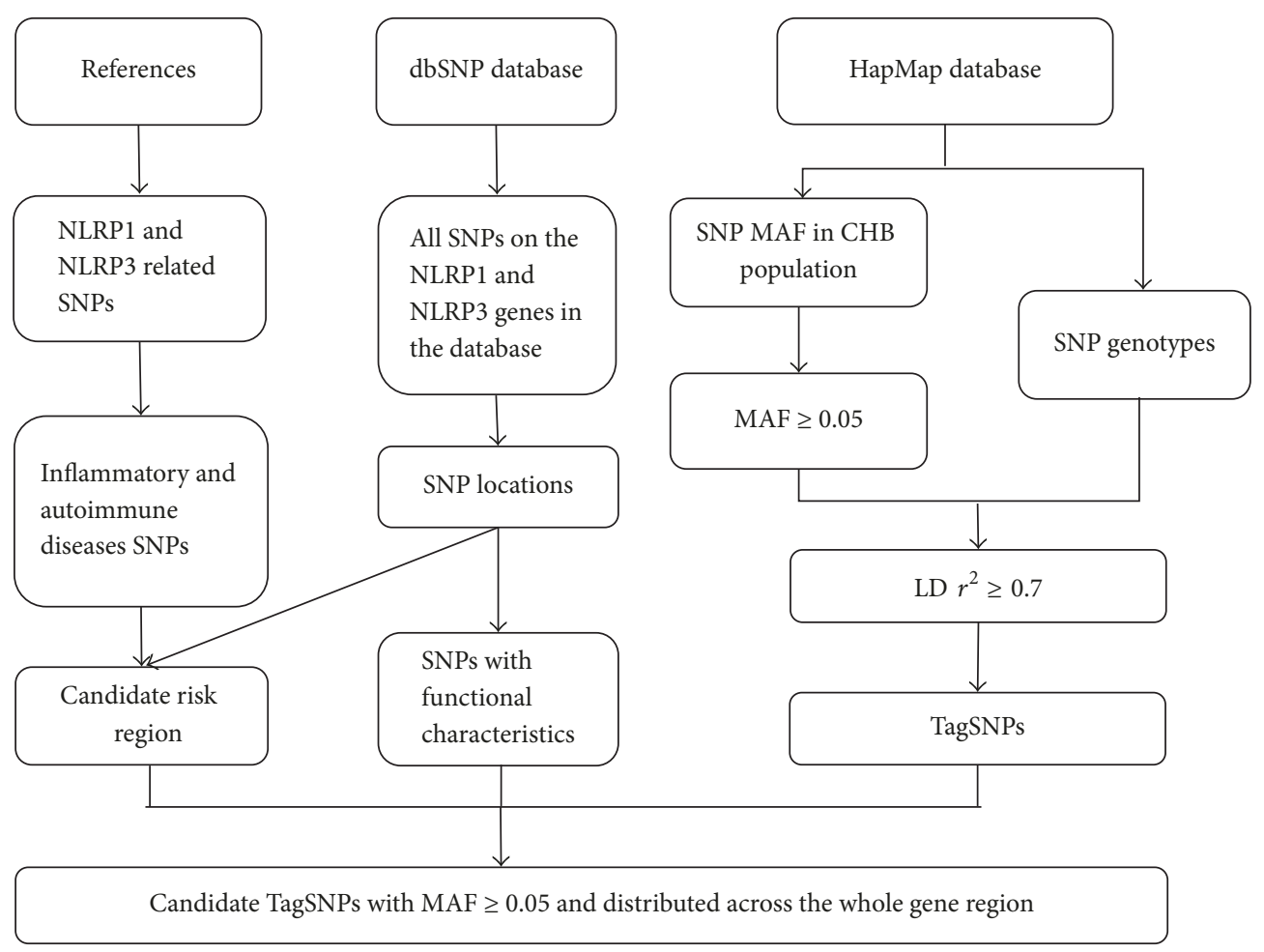

FIGURE 1: Flow chart depicting the process of SNP selection.

$2 \mu \mathrm{L}$ purified PCR products, and $6 \mu \mathrm{L}$ ddH2O. The thermal conditions were as follows: 38 cycles of $94^{\circ} \mathrm{C}$ for $1 \mathrm{~min}, 56^{\circ} \mathrm{C}$ for $4 \mathrm{~min}$, and holding at $4^{\circ} \mathrm{C}$. The diluted ligation product $(0.5 \mu \mathrm{L})$ was mixed with $0.5 \mu \mathrm{L}$ Liz500 Size Standard and $9 \mu \mathrm{L} \mathrm{Hi}-\mathrm{Di}$, denatured at $95^{\circ} \mathrm{C}$ for $5 \mathrm{~min}$, and then loaded in the ABI3730XL sequencer. The raw data were analyzed using GeneMapper 4.1 software (Applied Biosystems, Foster City, CA, USA). The SNPs were identified according to different extension lengths of the $3^{\prime}$ terminal. We used different fluorescent labels of the allele-specific oligonucleotide probe to distinguish the specific alleles of each SNP.

Finally, to ensure the quality of the data, genotyping was performed by independent staff blinded to the case/control status of the samples, using ddH2O as the negative control for each reaction. A random DNA sample accounting for $5 \%$ of the total was genotyped again to determine the reproducibility.

2.5. Statistical Analysis. Demographic characteristics between the case and control groups (e.g., age, gender, stage, age of onset, severity, and family history) were evaluated by SPSS version 19.0. The distribution of detected genotypes, alleles, and conformation with Hardy-Weinberg equilibrium (HWE, evaluated at <0.001) were performed using Haploview 4.0. Four genetic models were adopted based on the allele frequencies of each locus in the cases to further evaluate differences in genotype distributions: additive, dominant, recessive, and heterozygous models. The allele and genotype frequencies were statistically compared between the cases and controls for each genetic model. The chi-squared test was used to establish the associations of NLRP1 and NLRP3 polymorphisms between cases and controls. Single SNP association analyses were conducted using SNPtest (wtccc) (http://mathgen.stats.ox.ac.uk/genetics_software/snptest/ snptest.html). The association model (additive, dominant, recessive, and heterozygous) of each allele was tested using logistic regression.

The statistical significance was defined as $p<0.05$ for a single test. Subsequently, we used the permutation method to perform the multiple test correction. For each SNP, we kept the population number of case and control groups unchanged, but disturbed the genotype 10,000 times to obtain 10,000 chi-square $p$ values for the disturbed samples. Then, we defined the $p^{\prime}$ value equivalent to the distribution of the original $p$ calculated from the true data in the simulated $p$ values. The linkage disequilibrium (LD), haplotype inference, and haplotype-based association analyses were carried out using Haploview tools. The LD $\left(r^{2}\right)$ threshold was 0.8 and $p<0.05$ was identified as the statistically significant LD. Finally, we estimated the sample size to confirm the reliability of the data results.

\section{Results}

3.1. Samples and Description of the Genotyping Data. In total, 1152 subjects that participated in the study were successfully genotyped. The case and control groups were effectively ageand gender-matched ( $p=0.20$ and $p=0.24$, resp.), and there was no significant difference $(p>0.05)$ between NLRP1 and NLRP3 genotypes according to age at disease 
TABLE 1: Characteristics of the study participants.

\begin{tabular}{lccc}
\hline Characteristic & Cases & Controls & $p$ \\
\hline Gender, $n(\%)$ & & & 0.062 \\
$\quad$ Male & $332(61.5 \%)$ & $342(55.9 \%)$ & \\
Female & $208(38.5 \%)$ & $270(44.1 \%)$ & \\
Age, mean \pm SD & $44.23 \pm 12.44$ & $45.47 \pm 12.73$ & 0.238 \\
Age at onset, $n(\%)$ & & & \\
$\quad \leq 40$ years & $439(81.3 \%)$ & & \\
$>40$ years & $101(18.7 \%)$ & & \\
PASI, $n(\%)$ & & & \\
$\leq 10$ & $446(82.6 \%)$ & & \\
$>10$ & $94(17.4 \%)$ & & \\
Family history, $n(\%)$ & & \\
Yes & $203(37.6 \%)$ & \\
No & $337(62.4 \%)$ & & \\
\hline
\end{tabular}

$n$, number; PASI, psoriasis area and severity index.

onset ( $\leq 40$ versus $\geq 40$ years) or severity or family history. The blinded genotyping concordance rate was $100 \%$, and no discrepancies were identified in the randomly selected (5\%, $n=58)$ samples. The characteristics of the participants are provided in Table 1 . In the case group, most PsV patients were in relatively mild condition ( $82.6 \%$ with PASI (psoriasis area and severity index) $\leq 10$ and $17.4 \%$ with PASI $\geq 10$ ).

In the 1152 quality control samples, the genotype reproducibility was $100 \%$. No SNP revealed evidence of Mendelian genetic error, and none of the polymorphisms deviated from the HWE expectation in either cases or controls.

3.2. Single SNP Association Analysis. Initially, we selected 32 SNPs in NLRP1 with MAF $\geq 0.05$ in CHB population, which contains 5 linkage disequilibrium modules. Combining with the SNPs mentioned in the literature and function characteristics of theses SNPs, we chose a representative SNP from each module, and finally 4 SNPs were identified. Similarly, we selected 116 SNPs in NLRP3 with a MAF $\geq 0.05$ in CHB population, which contains 12 linkage disequilibrium modules. Combining with the SNPs mentioned in the literature and SNPs function characteristics, we chose a representative SNP from each module, and finally 4 SNPs were chosen.

The human NLRP1 gene is $83.1 \mathrm{~kb}$ long, located on chromosome 17p13.2. The human NLRP3 gene is $33.0 \mathrm{~kb}$ long, located on chromosome 1q44. The allele and genotype frequencies of the eight SNPs in patients and controls are summarized in Tables 2-4. For the rs3806265 SNP, the risk allele $\mathrm{T}$ was present in $56.5 \%$ of patients and $50.7 \%$ of controls. For the rs10754557 SNP, the risk allele A was present in 74.1\% of patients and $69.1 \%$ of controls. The genotype distribution of the rs3806265 SNP was significantly different between the cases and controls $\left(p=0.0451 ; p^{\prime}=0.0474 ; \mathrm{OR}=\right.$ $0.791)$. Additionally, in the recessive model (AA + AG versus GG), the genotype distribution of the rs10754557 SNP was significantly different between the cases and controls ( $p=$ $0.0344 ; p^{\prime}=0.0352$; OR $=1.277$ ). We calculated the power of the genotype distribution using the PASS11 and found that the power of the genotype distribution of the rs3806265 SNP was 0.68420 and that of the rs10754557 SNP in the recessive model was 0.84836 . However, there were no significant differences between the groups in either the genotype or allele frequencies for the SNPs rs8079034 (intron of NLRP1), rs11651270 (missense of NLRP1), rs11657747 (missense of NLRP1), rs878329 (31 kb $5^{\prime}$ of NLRP1), rs7512998 (intron of NLRP3), and rs10733113 (10 kb 3' of NLRP3) (all $p>0.05)$.

3.3. Haplotype-Based Association Analysis. Haploview analysis revealed that there are 6 and 7 haplotype blocks existing on NLRP1 and NLRP3, respectively, with the estimated frequency of more than 0.01 . None of the 15 haplotypes were significantly associated with PsV risk $(p>0.05)$ (see Tables S3-S4 in the Supplementary Materials for the haplotypes information). As rs3806265 and rs10754557 SNPs in NLRP3 are significantly associated with $\mathrm{PsV}$, we also performed a haplotype-based association study specifically regarding the two SNPs. For the 4 blocks with the threshold of $>0.01$ (shown in Table 5), the most common was TA haplotype, and it was significantly associated with $\operatorname{PsV}\left(\chi^{2}=4.529 ; p=0.033\right)$.

\section{Discussion}

Through this case-control study, we investigated the possible association between NLRP1 and NLRP3 polymorphisms and $\mathrm{PsV}$ in Chinese Han population, by studying eight candidate SNPs. We observed that rs3806265 and rs10754557 in NLRP3 were associated with PsV.

Regarding $\mathrm{PsV}$, a common and complex immunemediated inflammatory disease, genetic factors have been shown to play a critical role in its pathogenesis [24], including numerous genetic variants related to innate immunity [25]. Given the key role of the NLRP family in innate immune regulation [18], we explored the association between SNPs in NLRP1 and NLRP3 and PsV.

In this study, rs3806265 and rs10754557 in NLRP3 are associated with susceptibility to PsV. These results are partly consistent with those of the Swiss studies [9, 12]; however, there are some differences worth mentioning, namely, the lack of association of rs10733113 and the SNPs in NLRP1 with PsV. However, an association study on Caucasians living in the UK showed that SNP rs3806265 in NLRP3 is associated with psoriatic juvenile idiopathic arthritis [26], which is consistent with our finding. This discrepancy might be related to the heterogeneity of this complex polygenetic hereditary disease. The effect of susceptibility loci on the risk of psoriasis development may change under environment-gene interactions. Samples stability may also affect the results of the study.

In order to explore the function of rs3806265 and rs10754557, we used the HaploReg version 4.1 database (http://www.broadinstitute.org/mammals/haploreg) to identify the potential functional annotation of the risk alleles. We selected the position weight matrices from TRANSFAC and JASPAR databases to examine the effects of the risk allele on transcription factor binding using HaploReg. We examined transcriptional regulatory features such as DNase sensitivity, histone modifications, and transcription factor binding in the University of California Santa Cruz (UCSC) Encyclopedia of DNA Elements (ENCODE) 
TABLE 2: Candidate SNPs location and frequency characteristics on NLRP1 and NLRP3 gene.

\begin{tabular}{|c|c|c|c|c|c|c|}
\hline \multirow{2}{*}{ Gene } & \multirow{2}{*}{ SNP } & \multirow{2}{*}{ Chromosome position } & \multirow{2}{*}{$\begin{array}{l}\text { Major/minor } \\
\text { allele }\end{array}$} & \multirow{2}{*}{$\begin{array}{l}\text { Risk } \\
\text { allele }\end{array}$} & \multicolumn{2}{|c|}{ Risk allele frequency } \\
\hline & & & & & Case & Control \\
\hline \multirow{4}{*}{$N L R P 1$} & rs8079034 & 5509041 & $\mathrm{C} / \mathrm{T}$ & $\mathrm{C}$ & $81.9 \%$ & $80.9 \%$ \\
\hline & rs11651270 & 5521757 & $\mathrm{~T} / \mathrm{C}$ & $\mathrm{C}$ & $24.3 \%$ & $23.7 \%$ \\
\hline & rs11657747 & 5541923 & $\mathrm{G} / \mathrm{A}$ & $\mathrm{G} / \mathrm{A}$ & - & - \\
\hline & rs878329 & 5649930 & $\mathrm{G} / \mathrm{C}$ & G & $82.6 \%$ & $81.5 \%$ \\
\hline \multirow{4}{*}{$N L R P 3$} & rs7512998 & 247419919 & $\mathrm{~T} / \mathrm{C}$ & $\mathrm{C}$ & $8.3 \%$ & $7.4 \%$ \\
\hline & rs3806265 & 247423034 & $\mathrm{~T} / \mathrm{C}$ & $\mathrm{T}$ & $56.5 \%$ & $50.7 \%$ \\
\hline & rs10754557 & 247435930 & $\mathrm{~A} / \mathrm{G}$ & A & $74.1 \%$ & $69.1 \%$ \\
\hline & rs10733113 & 247459055 & G/A & A & $6.1 \%$ & $4.9 \%$ \\
\hline
\end{tabular}

TABLE 3: The single SNP association studies result of NLRP1 on the risk of PV.

\begin{tabular}{|c|c|c|c|c|c|c|c|c|}
\hline Genotype & Cases $(n=540)$ & Controls $(n=612)$ & $p$ value & Adjusted $p$ value & Statistical model & $p$ & $p^{\prime}$ & OR $(95 \% \mathrm{CI})$ \\
\hline \multicolumn{9}{|l|}{ rs8079034 } \\
\hline $\mathrm{TT}$ & $16(3.0 \%)$ & $24(3.9 \%)$ & & & Additive & 0.8171 & 0.8374 & \\
\hline TC & $164(30.3 \%)$ & $186(30.4 \%)$ & & & Dominant & 0.5288 & 0.6392 & 1.066 \\
\hline $\mathrm{CC}$ & $360(66.7 \%)$ & $402(65.7 \%)$ & & & Recessive & 0.8040 & 0.8601 & $(0.792,1.435)$ \\
\hline \multirow[t]{2}{*}{$\mathrm{T} / \mathrm{C}$} & $196 / 884$ & $234 / 990$ & 0.6732 & 0.7022 & Heterozygous & 0.9955 & 0.9998 & \\
\hline & $18.1 \% / 81.9 \%$ & $19.1 \% / 80.9 \%$ & & & & & & \\
\hline \multicolumn{9}{|l|}{ rs11651270 } \\
\hline TT & $302(55.9 \%)$ & $360(58.8 \%)$ & & & Additive & 0.3893 & 0.3873 & \\
\hline $\mathrm{TC}$ & $214(39.6 \%)$ & $214(35.0 \%)$ & & & Dominant & 0.4828 & 0.5006 & 1.032 \\
\hline $\mathrm{CC}$ & $24(4.4 \%)$ & $38(6.2 \%)$ & & & Recessive & 0.3464 & 0.3666 & $(0.787,1.353)$ \\
\hline \multirow[t]{2}{*}{$\mathrm{T} / \mathrm{C}$} & $818 / 262$ & $934 / 290$ & 0.8204 & 0.8320 & Heterozygous & 0.2480 & 0.2711 & \\
\hline & $75.7 \% / 24.3 \%$ & $76.3 \% / 23.7 \%$ & & & & & & \\
\hline \multicolumn{9}{|l|}{ rs11657747 } \\
\hline GG & $526(97.4 \%)$ & $596(97.4 \%)$ & & & Additive & -- & 0.9999 & \\
\hline GA & $14(2.6 \%)$ & $16(2.6 \%)$ & & & Dominant & 0.9869 & 0.9998 & 0.992 \\
\hline AA & $0(0)$ & $0(0)$ & & & Recessive & -- & 0.9999 & $(0.357,2.753)$ \\
\hline \multirow[t]{2}{*}{$\mathrm{G} / \mathrm{A}$} & $1066 / 14$ & $1208 / 16$ & 0.9869 & 0.9998 & Heterozygous & 0.9870 & 0.9999 & \\
\hline & $98.7 \% / 1.3 \%$ & $98.7 \% / 1.3 \%$ & & & & & & \\
\hline \multicolumn{9}{|l|}{ rs878329 } \\
\hline GG & $364(67.4 \%)$ & $400(65.3 \%)$ & & & Additive & 0.8724 & 0.8946 & \\
\hline GC & $164(30.4 \%)$ & $198(32.4 \%)$ & & & Dominant & 0.60365 & 0.6660 & 0.931 \\
\hline $\mathrm{CC}$ & $12(2.2 \%)$ & $14(2.3 \%)$ & & & Recessive & 0.9580 & 0.9999 & $(0.688,1.259)$ \\
\hline \multirow[t]{2}{*}{$\mathrm{G} / \mathrm{C}$} & $892 / 188$ & $998 / 226$ & 0.62939 & 0.6359 & Heterozygous & 0.6089 & 0.6540 & \\
\hline & $82.6 \% / 17.4 \%$ & $81.5 \% / 18.5 \%$ & & & & & & \\
\hline
\end{tabular}

$N$, number; $p$, model-based statistical $p$ value; $p^{\prime}, p$ value adjusted by permutation; OR, odds ratio (we used the major allele as the reference allele to calculate the OR); $95 \%$ CI, $95 \%$ confidence interval.

database (https://www.encodeproject.org/). Available HaploReg data indicated that rs 3806265 is located in intron region and histone marks (H3K4mel_Enh) are detected at the locus based on histone modification feature data in many immunerelated cell lines (primary $\mathrm{T}$ helper memory cells from peripheral blood, primary T CD8+ naive cells from peripheral blood, and primary monocytes from peripheral blood), suggesting that this locus may function as an enhancer in the immune-related system. For rs10754557, we found that it is a trans-eQTL for NLRP3 in whole blood according the HaploReg dataset, suggesting that the effects of rs10754557 and NLRP3 expression on disease developing risk may differ for individuals with different genotype.

It is worth mentioning that, in this study, most PsV patients are in relatively mild condition, which may be due to poor tolerance of patients with PsV, without waiting for the serious development of timely treatment. However, we did not find significant statistical differences between the NLRPI 
TABLE 4: The single SNP association studies result of NLRP3 on the risk of PV.

\begin{tabular}{|c|c|c|c|c|c|c|c|c|}
\hline Genotype & Cases $(n=540)$ & Controls $(n=612)$ & $p$ value & Adjusted $p$ value & Statistical model & $p$ & $p^{\prime}$ & OR $(95 \% \mathrm{CI})$ \\
\hline \multicolumn{9}{|l|}{ rs7512998 } \\
\hline TT & $450(83.3 \%)$ & $524(85.6 \%)$ & & & Additive & 0.3710 & 0.4540 & \\
\hline $\mathrm{TC}$ & $90(16.7 \%)$ & $86(14.1 \%)$ & & & Dominant & 0.4488 & 0.4969 & 1.146 \\
\hline $\mathrm{CC}$ & $0(0.0 \%)$ & $2(0.3 \%)$ & & & Recessive & -- & 0.9998 & $(0.745,1.762)$ \\
\hline \multirow[t]{2}{*}{$\mathrm{T} / \mathrm{C}$} & $990 / 90$ & $1134 / 90$ & 0.5235 & 0.5760 & Heterozygous & 0.3846 & 0.4227 & \\
\hline & $91.7 \% / 8.3 \%$ & $92.6 \% / 7.4 \%$ & & & & & & \\
\hline \multicolumn{9}{|l|}{ rs3806265 } \\
\hline $\mathrm{TT}$ & $166(30.8 \%)$ & $156(25.5 \%)$ & & & Additive & 0.1191 & 0.1157 & \\
\hline TC & $278(51.5 \%)$ & $308(50.3 \%)$ & & & Dominant & 0.1614 & 0.1613 & 0.791 \\
\hline $\mathrm{CC}$ & $96(17.7 \%)$ & $144(24.2 \%)$ & & & Recessive & 0.0595 & 0.0629 & $(0.627,0.998)$ \\
\hline \multirow[t]{2}{*}{$\mathrm{T} / \mathrm{C}$} & $610 / 470$ & $620 / 604$ & $0.0451^{*}$ & $0.0474^{*}$ & Heterozygous & 0.7821 & 0.8072 & \\
\hline & $56.5 \% / 43.5 \%$ & $50.7 \% / 49.3 \%$ & & & & & & \\
\hline \multicolumn{9}{|l|}{ rs10754557 } \\
\hline GG & $40(7.4 \%)$ & $52(8.5 \%)$ & & & Additive & 0.1054 & 0.1019 & \\
\hline GA & $200(37.0 \%)$ & $274(44.8 \%)$ & & & Dominant & 0.6298 & 0.6516 & 1.277 \\
\hline $\mathrm{AA}$ & $300(55.6 \%)$ & $286(46.7 \%)$ & & & Recessive & $0.0344^{*}$ & $0.0352^{*}$ & $(0.987,1.652)$ \\
\hline \multirow[t]{2}{*}{ G/A } & $360 / 800$ & $378 / 846$ & 0.0614 & 0.0655 & Heterozygous & 0.0595 & 0.0577 & \\
\hline & $25.9 \% / 74.1 \%$ & $30.9 \% / 69.1 \%$ & & & & & & \\
\hline \multicolumn{9}{|l|}{ rs10733113 } \\
\hline GG & $474(87.8 \%)$ & $552(90.2 \%)$ & & & Additive & -- & 0.9999 & \\
\hline GA & $66(12.2 \%)$ & $60(9.8 \%)$ & & & Dominant & 0.3540 & 0.4282 & 1.263 \\
\hline AA & $0(0.0 \%)$ & $0(0.0 \%)$ & & & Recessive & -- & 0.9998 & $(0.760,2.100)$ \\
\hline \multirow[t]{2}{*}{ G/A } & $1014 / 66$ & $1164 / 60$ & 0.3540 & 0.4282 & Heterozygous & 0.3540 & 0.4282 & \\
\hline & $93.9 \% / 6.1 \%$ & $95.1 \% / 4.9 \%$ & & & & & & \\
\hline
\end{tabular}

$N$, number; $p$, model-based statistical $p$ value; $p^{\prime}, p$ value adjusted by permutation; OR, odds ratio (we used the major allele as the reference allele to calculate the OR); $95 \% \mathrm{CI}, 95 \%$ confidence interval; $*$ indicates the significant association.

TABLE 5: The haplotype-based association study of rs3806265 and rs10754557 SNPs in NLRP3 gene.

\begin{tabular}{|c|c|c|c|c|c|}
\hline Haplotype & Freq. & Case, control ratio counts & Case, control freqs. & $\chi^{2}$ & $p$ \\
\hline $\mathrm{TA}$ & $50.7 \%$ & $291.9: 248.1,292.4: 319.6$ & $54.1 \%, 47.8 \%$ & 4.529 & 0.0333 \\
\hline CG & $25.9 \%$ & $126.9: 413.1,171.4: 440.6$ & $23.5 \%, 28.0 \%$ & 3.031 & 0.0817 \\
\hline CA & $20.7 \%$ & $108.1: 431.9,130.6: 481.4$ & $20.0 \%, 21.3 \%$ & 0.307 & 0.5797 \\
\hline TG & $2.7 \%$ & $13.1: 526.9,17.6: 594.4$ & $2.4 \%, 2.9 \%$ & 0.227 & 0.6335 \\
\hline
\end{tabular}

Freq., frequency; $\chi^{2}$, chi-square; $p, p$ value.

and NLRP3 genotypes and the two groups of different severity of psoriasis, suggesting that both groups of different severity contribute to the overall significance. This is consistent with the findings of Europeans $[9,12]$.

This work was independently done by our team, including the sample collection, SNP genotyping, and statistical analysis. There are some limitations to our study. First, we analyze a single ethnic population in a single region, which inevitably leads to a certain degree of selection bias; the result may not be representative for other human races. Thus, to completely elucidate the roles of NLRP1 and NLRP3 in PsV, further studies in ethnically diverse populations are required to confirm or refute our findings. Second, with reference to previous literatures, in this study, we only included three clinical features to support the research samples. In future studies, we should consider more suspicious factors and make more detailed analysis so that our research can provide more value for the understanding of PsV. Additionally, subsequent functional evaluations are required to verify the functional prediction for the SNPs.

\section{Conclusions}

Taken together, this study investigated the association between NLRP1 and NLRP3 polymorphisms and the risk of $\mathrm{PsV}$ in the Chinese Han population. Of the eight candidate SNPs investigated in NLRP1 and NLRP3, two SNPs in NLRP3 (rs3806265 and rs10754557) were found to be significantly associated with PsV in the Chinese Han population. Overall, these findings supported the role of innate immunity in the pathogenesis of PsV and indicated that NLRP3 polymorphisms may be a useful genetic marker or causative genetic 
factor in PsV development. Moreover, NLRP3 may be a potential therapeutic target for PsV.

\section{Data Availability}

According to the informed consent signed by the volunteers, the aggregated SNP genotype frequency and allele frequency data in the manuscript can be available freely to the academic community. The SNP genotype and the clinical information can be obtained by e-mail after security audit.

\section{Conflicts of Interest}

The authors declare that there are no conflicts of interest regarding the publication of this paper.

\section{Acknowledgments}

The authors are most grateful to the members of all the families who have so willingly participated in this study. This work was supported by the National Natural Science Foundation of China (no. 81371725).

\section{Supplementary Materials}

Tables S1-S2: primers and probes displays for PCR and imLDR. Tables S3-S4: haplotype analysis. Table S1: primers used for the genotyping of NLRP1 and NLRP3. Table S2: iMLDR probe sequences. Table S3: the haplotype-based association study of NLRP1 gene polymorphisms. Table S4: the haplotype-based association study of NLRP3 gene polymorphisms. (Supplementary Materials)

\section{References}

[1] N. Etemadi, M. Chopin, H. Anderton et al., "Correction: TRAF2 regulates TNF and NF- $\kappa \mathrm{B}$ signalling to suppress apoptosis and skin inflammation independently of Sphingosine kinase 1," eLife, vol. 6, Article ID e10592, 2017.

[2] S. K. Mahil, F. Capon, and J. N. Barker, "Update on psoriasis immunopathogenesis and targeted immunotherapy," Seminars in Immunopathology, vol. 38, no. 1, pp. 11-27, 2016.

[3] X. Yin, H. Cheng, Y. Lin et al., "A weighted polygenic risk score using 14 known susceptibility variants to estimate risk and age onset of psoriasis in Han Chinese," PLoS ONE, vol. 10, no. 5, Article ID e0125369, 2015.

[4] L. C. Tsoi, P. E. Stuart, C. Tian et al., "Large scale meta-analysis characterizes genetic architecture for common psoriasis associated variants," Nature Communications, vol. 8, Article ID 15382, 2017.

[5] S. Grandemange, E. Sanchez, P. Louis-Plence et al., "A new autoinflammatory and autoimmune syndrome associated with NLRP1 mutations: NAIAD (NLRP1- associated autoinflammation with arthritis and dyskeratosis)," Annals of the Rheumatic Diseases, vol. 76, no. 7, pp. 1191-1198, 2017.

[6] H. Wang, P. Xu, D. Liao et al., "Association between NLPR1, NLPR3, and P2X7R gene polymorphisms with partial seizures," BioMed Research International, vol. 2017, Article ID 9547902, 6 pages, 2017.
[7] G. V. Paramel, A. Sirsjö, and K. Fransén, "Role of genetic alterations in the NLRP3 and CARD8 genes in health and disease," Mediators of Inflammation, vol. 2015, Article ID 846782, 10 pages, 2015.

[8] K. Schroder and J. Tschopp, “The inflammasomes,” Cell, vol. 140, no. 6, pp. 821-832, 2010.

[9] A.-K. Ekman, D. Verma, M. Fredrikson, C. Bivik, and C. Enerbäck, "Genetic variations of NLRP1: susceptibility in psoriasis," British Journal of Dermatology, vol. 171, no. 6, pp. 15171520, 2014.

[10] M. Ben Hamad, F. Cornelis, S. Marzouk et al., "Association study of CARD8 (p.C10X) and NLRP3 (p.Q705K) variants with rheumatoid arthritis in French and Tunisian populations," International Journal of Immunogenetics, vol. 39, no. 2, pp. 131136, 2012.

[11] M. L. Salskov-Iversen, C. Johansen, K. Kragballe, and L. Iversen, "Caspase-5 expression is upregulated in lesional psoriatic skin," Journal of Investigative Dermatology, vol. 131, no. 3, pp. 670-676, 2011.

[12] M. Carlström, A.-K. Ekman, S. Petersson, P. Söderkvist, and C. Enerbäck, "Genetic support for the role of the NLRP3 inflammasome in psoriasis susceptibility," Experimental Dermatology, vol. 21, no. 12, pp. 932-937, 2012.

[13] X. Dai, K. Sayama, M. Tohyama et al., "Mite allergen is a danger signal for the skin via activation of inflammasome in keratinocytes," The Journal of Allergy and Clinical Immunology, vol. 127, no. 3, pp. 806.e4-814.e4, 2011.

[14] Y. Li, H. Cheng, X.-B. Zuo et al., "Association analyses identifying two common susceptibility loci shared by psoriasis and systemic lupus erythematosus in the Chinese Han population," Journal of Medical Genetics, vol. 50, no. 12, pp. 812-818, 2013.

[15] A. Hinks, P. Martin, S. D. Thompson et al., "Autoinflammatory gene polymorphisms and susceptibility to UK juvenile idiopathic arthritis," Pediatric Rheumatology, vol. 11, no. 1, article no. 14, 2013.

[16] J. Sui, H. Li, and Y. Fang, "NLRP1 gene polymorphism influences gene transcription and is a risk factor for rheumatoid arthritis in han chinese," Arthritis \& Rheumatology, vol. 64, no. 3, pp. 647-654, 2012.

[17] Q. Zhang, H. W. Fan, J. Z. Zhang, Y. M. Wang, and H. J. Xing, "NLRP3 rs35829419 polymorphism is associated with increased susceptibility to multiple diseases in humans," Genetics and Molecular Research, vol. 14, no. 4, pp. 13968-13980, 2015.

[18] C. Bivik, D. Verma, M. C. Winge et al., "Genetic variation in the inflammasome and atopic dermatitis susceptibility," Journal of Investigative Dermatology, vol. 133, no. 10, pp. 2486-2489, 2013.

[19] D. Verma, C. Bivik, E. Farahani et al., "Inflammasome polymorphisms confer susceptibility to sporadic malignant melanoma," Pigment Cell \& Melanoma Research, vol. 25, no. 4, pp. 506-513, 2012.

[20] H. Ray-Jones, S. Eyre, A. Barton, and R. B. Warren, "One SNP at a Time: Moving beyond GWAS in Psoriasis," Journal of Investigative Dermatology, vol. 136, no. 3, pp. 567-573, 2016.

[21] L. D. Sun, H. Cheng, Z. X. Wang et al., "Association analyses identify six new psoriasis susceptibility loci in the Chinese population," Nature Genetics, vol. 42, pp. 1005-1009, 2010.

[22] Z. Zhao, M. Zhang, J. Ying et al., "Significance of genetic polymorphisms in long non-coding RNA AC079767.4 in tuberculosis susceptibility and clinical phenotype in Western Chinese Han population," Scientific Reports, vol. 7, no. 1, article no. 1163, 2017. 
[23] X. Hu, W. Peng, X. Chen et al., "No significant effect of ASAP1 gene variants on the susceptibility to tuberculosis in Chinese Population," Medicine (United States), vol. 95, no. 21, Article ID e3703, 2016.

[24] P. Qin, Q. Zhang, M. Chen et al., "Variant Analysis of CARD14 in a Chinese Han Population with Psoriasis Vulgaris and Generalized Pustular Psoriasis," Journal of Investigative Dermatology, vol. 134, no. 12, pp. 2994-2996, 2014.

[25] G. Shi, T. Wang, S. Li et al., "TLR2 and TLR4 polymorphisms in Southern Chinese Psoriasis Vulgaris patients," Journal of Dermatological Science, vol. 83, no. 2, pp. 145-147, 2016.

[26] T. G. Day, A. V. Ramanan, A. Hinks et al., "Autoinflammatory genes and susceptibility to psoriatic juvenile idiopathic arthritis," Arthritis \& Rheumatology, vol. 58, no. 7, pp. 2142-2146, 2008. 


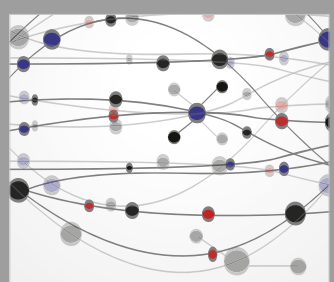

The Scientific World Journal
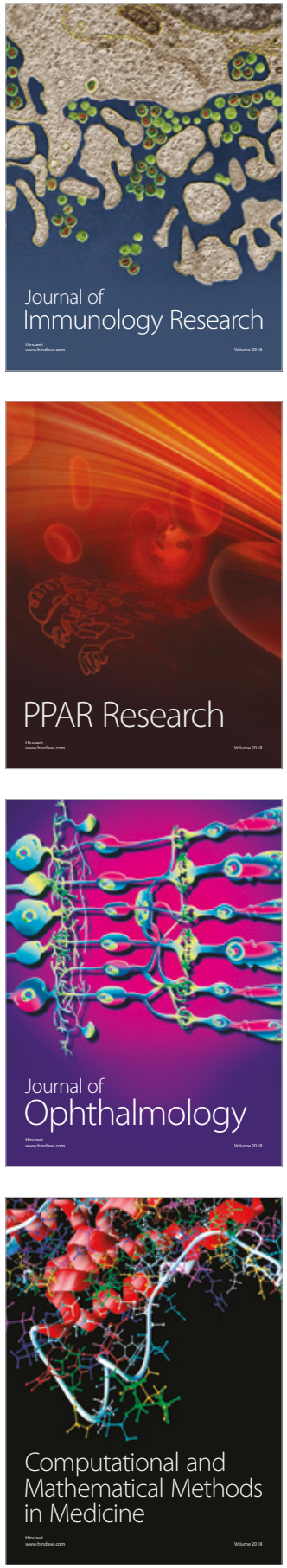

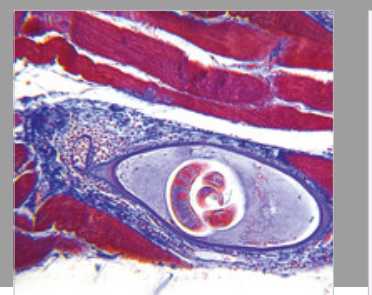

Gastroenterology Research and Practice

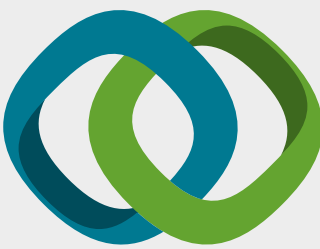

\section{Hindawi}

Submit your manuscripts at

www.hindawi.com
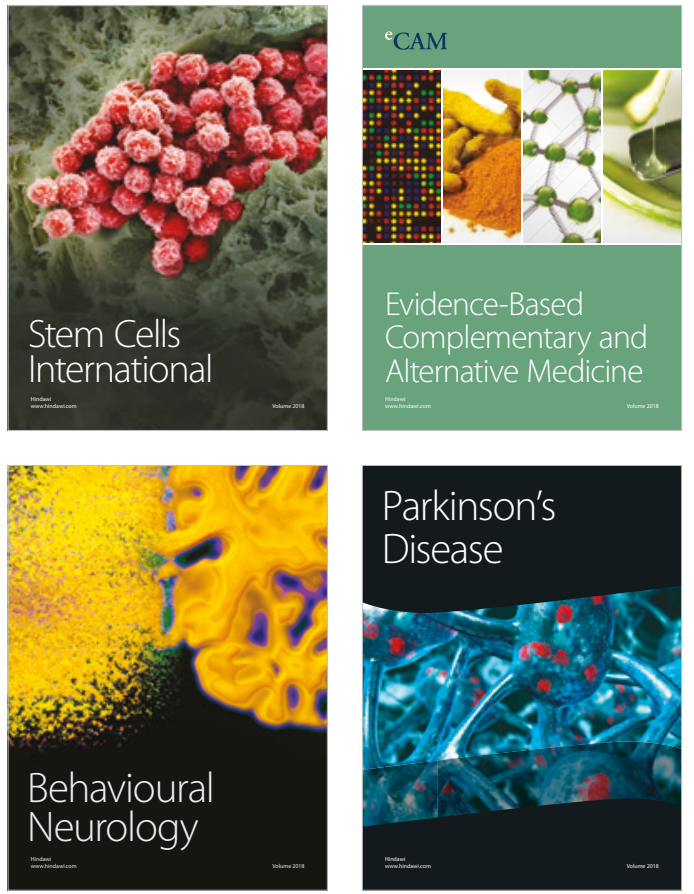

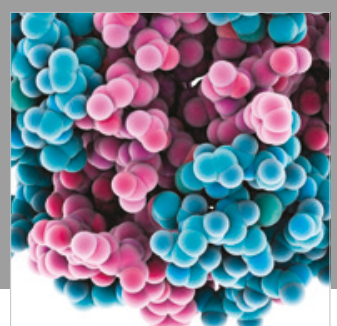

ournal of

Diabetes Research

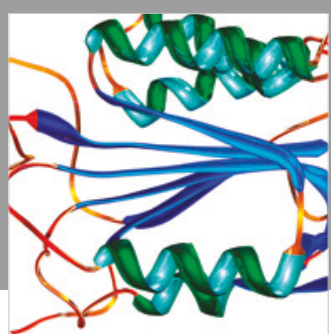

Disease Markers
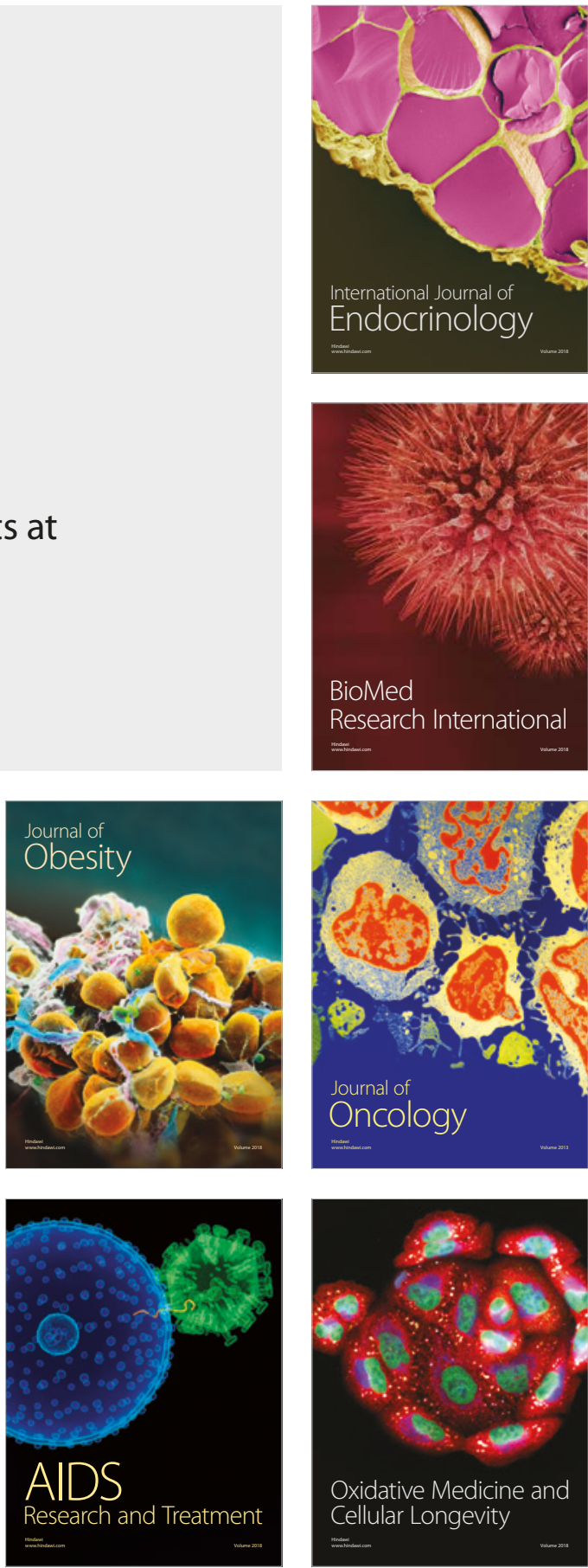